\title{
Integrated Public Grievance Redressal System (iPGRS) A Next-Gen Solution for a Next-Gen Railway
}

\author{
Kandukuri Raju, ${ }^{1, *}$, Shivam Gupta ${ }^{2}$ \\ ${ }^{1}$ Rail Domain Consultant, Travel, Transportation and Hospitality (TTH), Tata Consultancy Services (TCS), Pune, India \\ ${ }^{2}$ Rail Domain Business Analyst, Travel, Transportation and Hospitality (TTH), Tata Consultancy Services (TCS), New Delhi, India
}

Copyright $@ 2018$ by authors, all rights reserved. Authors agree that this article remains permanently open access under the terms of the Creative Commons Attribution License 4.0 International License

\begin{abstract}
Railways receive millions of service related complaints and grievances from the passengers annually through various channels (web-based, mobile app, social media, phone or paper based etc.). However, these channels are usually disparate, siloed, and heavy with manual processes. For many rail companies, there is no uniform business process or single platform visibility to address these grievances. The result is a high level complexity (multiple channels, differing processes and so on) that increases response times in addressing them. Railway companies in the Asian continent started to semi-automate their complaints processes but the process is not sufficiently robust to survive the large volumes expected in the future. Today, most railways employ a small 'grievance department' with people working in shifts 24x7 and manually handling thousands of complaints daily. Moreover, the penetration of smartphones is leading to a drastic increase in the volume of complaints, resulting in railways having to consider hiring more people. The result of this is two-fold $-\mathrm{i}$ ) added cost pressures due to increase in staffing and ii) reduction in revenues due to loss of passenger satisfaction. iPGRS is an omni-channel solution that helps railways overcome these challenges. iPGRS integrates grievances from multiple channels onto a single platform, and creates a Service Request (SR)-based workflow that enables monitoring of grievances resolutions. Concerned stakeholders in the grievance department receive these Service Requests as an action item in their workflow and it becomes their responsibility to properly address and close each request. Feedback related to a grievance resolution is integral to ensuring transparency and accountability in a system that handles large volumes of grievances. The iPGRS system also includes many automation techniques, like rule-based engine, text analytics and more, to speed up the whole process. iPGRS offers a 360-degree integrated dashboard for further analysis and service improvements. We developed a prototype and demonstrated this solution to a large passenger rail organization in Asia for their consideration.
\end{abstract}

Keywords Grievance, Automation, Workflow, Analytics, Dashboard

\section{Introduction}

Globally, railways make over 35 billion journeys every year. In 2016, global rail passenger traffic amounted to 2.8 trillion passenger-kilometers [1], with Asia, Oceania and the Middle East together accounting for more than three-fourths of the total. The outlook for the railway industry is quite optimistic, with one report predicting steady growth through to 2025. During this period, urban rail will grow the strongest, at an average 5 percent year-on-year, followed by the mainline passenger and freight segments [2].

The growth of urban rail is driven by the construction of new lines, particularly in China, whose network tripled between 2005 and 2015; countries in South and Central America have also contributed to this growth. The United Kingdom and some of the Arab nations are also investing in new lines. The United States, India and some Asian countries are planning high-speed rail networks.

The mainline passenger segment is finding pockets of growth in Asia as well as South and Central America. An interesting trend is the rejuvenation of rail travel in the mature markets of Western Europe and North America due to environmental regulations seeking to cut down on road traffic [2]. For example, Spain, which is one of the countries leaning towards public transport, is making fresh investments in railways between 2016 and 2025 [3].

This is welcome news for the railway industry, which, even today, is one of the most suitable modes of passenger transport given that it is cost-effective, environment-friendly and faster than many other types of land transportation [4]. Apart from being the cheapest and safest mode of long distance passenger transport, it is also emerging as a preferred mode of urban transportation 
amidst a rising population and overcrowding of urban areas, which is estimated to host 75 percent of the world's population by 2050 [5].

That being said, railways have to compete with airlines over long distances, and with buses, private cars, taxis and ride-sharing services, over short routes. This means trains must equal, or even exceed, the service and experience provided by these alternative modes of transport. A look at some of the biggest success stories in transportation highlight the importance of service: Southwest Airlines, which had the highest customer satisfaction among low-cost airlines in 2017 [6], inspires great loyalty among customers, and despite a late entry, has risen to the top position and presently occupies a market share of nearly 20 percent [7]. And what more can be said about Uber, which has become a metaphor for high quality service and experience?

In the consumption lifecycle, a key moment of truth is when a customer reaches out to complain about a product, service or experience. Recognizing that, the Social Customer Care Team at Southwest Airlines tries to resolve all queries received through social channels speedily without escalating the issue or passing the buck to another service channel [8]. Like airlines, the railways sector is also focusing on enhancing customer experience by providing passengers with real time journey information, multi-channel booking platforms, on-board entertainment and more. But if rail transportation has to lead other modes of transport, it must also establish a highly efficient, reliable and responsive complaint handling mechanism. The good news is that, with the availability of new age technologies and sophisticated IT systems, a railway can easily establish such solutions within its ecosystem.

\section{Need for an Integrated Real Time Grievance Redressal Solution}

For an industry that has digitized its ticketing processes quite successfully, the railways have a surprisingly outdated grievance handling mechanism. In India, which boasts one of the largest networks in the world, rail passengers air millions of grievances using a plethora of channels - phone, email, web, mobile app, snail mail and social media. These channels are disparate, work in silos and follow different (mostly manual) processing mechanisms. The complaints are consolidated by a small central unit that reroutes them manually to the right stakeholders for corrective action. However, this arrangement involves some amount of delay as well as considerable manpower costs. Moreover, once a complaint is passed on, there is no mechanism to see if it has been resolved successfully, nor is there any way of knowing:

a. The number of unresolved complaints

b. The number of resolved complaints

c. The amount of time it took to resolve a set of complaints

d. Responses to specific customer feedback

This lack of visibility among railway operators diminishes both the quality and speed of response.

Railway operators in the Asian continent have partially automated their service desks, in particular to be able to handle complaints posted via Twitter - a channel that's rapidly gaining in popularity. However, the infrastructure is typically not robust or scalable enough to survive the heavy workload expected in the future. Even in a mature market like the United Kingdom, passengers are quite disillusioned with the service on trains. In 2016, complaints shot up 31 percent to touch 2 million. A spokesperson for "Campaign for Better Transport" has demanded a review of the complaints system to improve its performance and asked regulators to ensure that complaints receive proper attention and action, rather than lip service [9].

Clearly, there is a strong need in several countries for a robust, integrated public grievance redressal system that can automate the gathering and rerouting of complaints from all channels to complete those processes in (near) real time, offer a consolidated view of all complaints irrespective of channel (online, mobile, Twitter, phone, etc.) - on a single platform, provide a mechanism to close the feedback loop, and enable better governance and monitoring of passenger complaint handling.

A system such as this would enhance service quality and customer satisfaction by not only improving response times and reliability, but also eliminating some of the issues that might arise from the attitude and behavior of service staff. Another important benefit is cost savings as a result of replacing expensive manpower with automation. But above all, an effective grievance redressal mechanism goes a long way in building trust and loyalty among passengers. Figure 1 depicts the process and challenges associated with typical current passenger grievance redressal systems. 


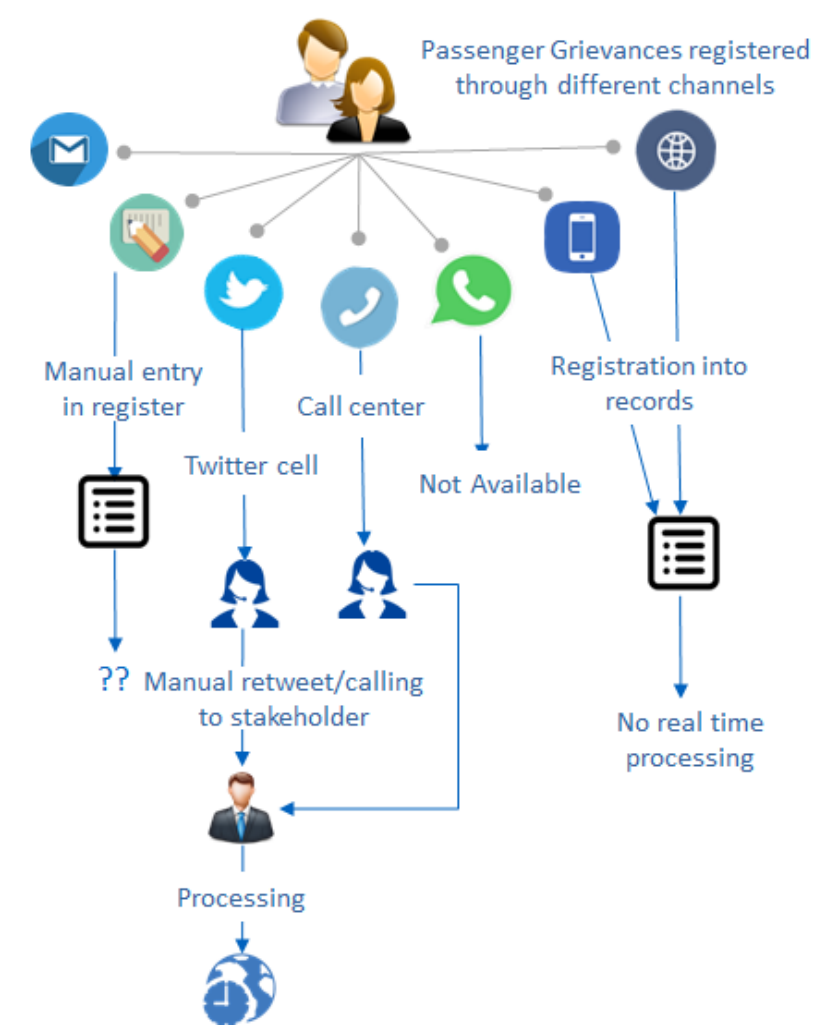

Figure 1. A typical Passenger Grievance Redress System in operation today

\section{Overview of an Integrated Public Grievance Redressal System, or iPGRS}

Integrated Public Grievance Redressal System (iPGRS) is an omni-channel (online and mobile) solution that reduces lead times required to address passenger grievances (depicted in Figure 2). The solution achieves this by:

a. Integrating all the channels where complaints originate

b. Streamlining the end-to-end business processes and

c. Providing a 360-degree, integrated view of passenger grievances across the entire railway network to drive improved analyses, service levels and accountability

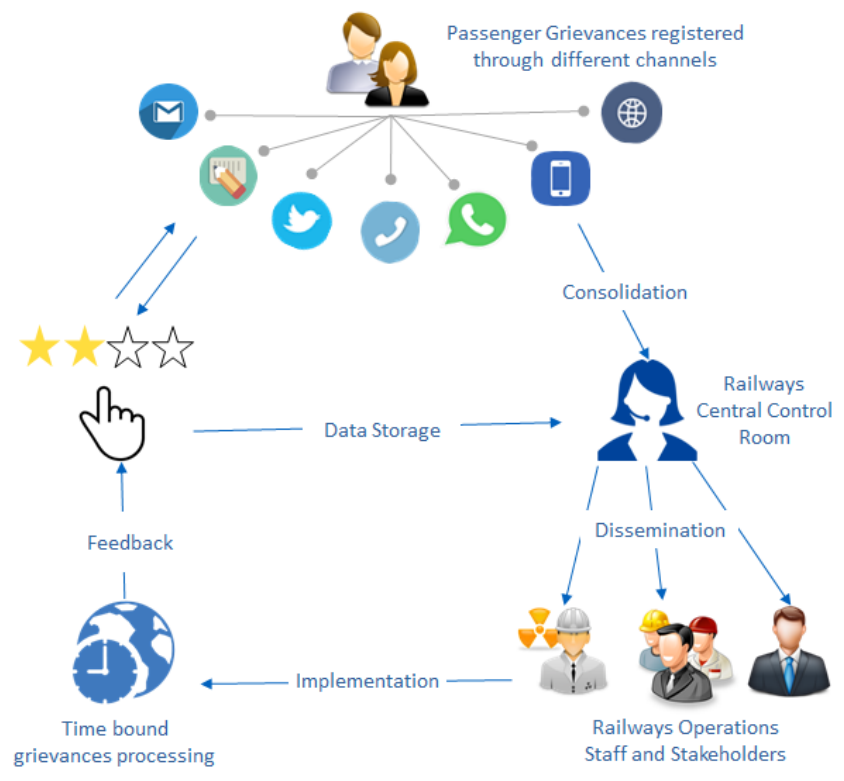

Figure 2. Overview of proposed iPGRS 


\section{Components of iPGRS Solution}

The following five major components describe the iPGRS solution in detail

1. Consolidation: This component registers and consolidates grievances from various channels onto a single platform or database. It receives grievances from channels like Phone (IVRS, voice), Mobile application, Web application, paper based, email, social media (twitter, fb, etc.) and instant messaging (whatsapp, imessage, chatbot). The content of every complaint is converted into a standardized electronic form. Grievances received over channels such as mobile application, web application are obviously easier to capture, and rail organizations can extract information directly as registered by passenger. However complaints sent via channels such as phone call, email, social media communications, instant messaging, would be conversational in nature where passenger would be registering grievances in verbal or text format. For these types of channels, a natural language processing tool is used to extract the information from the text, into the standardized format. Paper based complaints also aren't left out - they are scanned and converted to the same electronic format. The standardized format is such that it takes minimum required inputs from the passenger and at same time understands the need and automates the workflow process. For eg: information like passenger journey details, grievance type, place of incidence and passenger contact details may be required to initiate the grievance. Once a grievance is registered, it is streamlined and integrated. The system uses business logic to verify if each complaint provides all the necessary details and information (such as train number, PNR (Passenger Name Record) number of ticket etc.) required for taking action, and highlights gaps (if any) in information. Where there is a gap, the concerned passenger is contacted through the same channel or the most logical one for example, those posting grievances via the website are contacted using email), to fill in the missing details. While this process does involve some manpower - to attend to phone calls or work a chat room, for example - it is very much possible to replace it with an automated solution, such as an Interactive Voice Response (IVR) or chatbot.

2. Dissemination: Complaints that are complete in every respect have to be disseminated to the concerned departments to be resolved by the operational crew. This is how it happens: iPGRS assigns a Service Request (SR) number to each complaint to uniquely identify it. Following business rules, it further sanitizes the list of grievances by classifying (housekeeping, electrical, food and beverage, etc.), de-duplicating (clubbing all grievances about the same problem into one batch - for example, all complaints about non-functioning air conditioning inside a particular coach will be clubbed together) and prioritizing genuine complaints, while weeding out fake ones. Now it is time to disseminate the work to the right people. The system has predefined workflows based on the type, nature or priority of grievance, which is used to route work to the person responsible, such as the train manager or station staff. All throughout, there is real-time visibility into the various actions and events unfolding in the case of each grievance.

3. Implementation: While remedial actions are usually implemented manually, iPGRS ensures this is done in a timely manner. It does this with the help of various SLA-driven parameters based on the nature and urgency of the problem. For instance, a problem that could impact passenger health or safety has to adhere to more exacting standards than a problem related to, for instance, passenger comfort. Apart from ensuring timely resolution, the system also enforces accountability. Unlike in the past, when many instructions were issued over the phone and were never recorded, iPGRS ensures there is now complete traceability for each and every complaint and its ensuing workflow. Every workflow is monitored round-the-clock and if it looks like one might overshoot the prescribed SLA, communication is initiated proactively with the concerned stakeholder.

4. Feedback: The inability to close the passenger feedback loop satisfactorily is a major drawback of current railway complaint handling systems. The feedback component of iPGRS addresses this lacuna by providing a mechanism to capture passenger feedback via the channel of original contact once remedial action has been taken. Service requests that have been fulfilled to the passengers' satisfaction are closed while unaddressed grievances are rerouted for further attention and even escalated if need be.

5. Analytics: Each of the processes mentioned above generate large quantities of data that can be mined for valuable insights. The analytics component of iPGRS processes a variety of data-complaints received, action taken, feedback, and more- to deliver insights into passenger pain points and specific improvement areas, all on a user friendly management dashboard. For instance, it might flag the food quality on a particular train that has consistently been targeted by complaints. While the first four components of iPGRS work at a tactical level and cater to operational staff, the analytics 
component alleviates an important pain point of railway company executives, who are often hampered by the lack of a holistic picture of grievances and their remediation status at any given time. By providing them with the visibility they previously lacked, the system enables railway company managements to take better strategic decisions.

Figure 3 presents the components of the iPGRS solution along with its basic characteristics and integrated system overview of the iPGRS solution with various components is depicted in Figure 4.

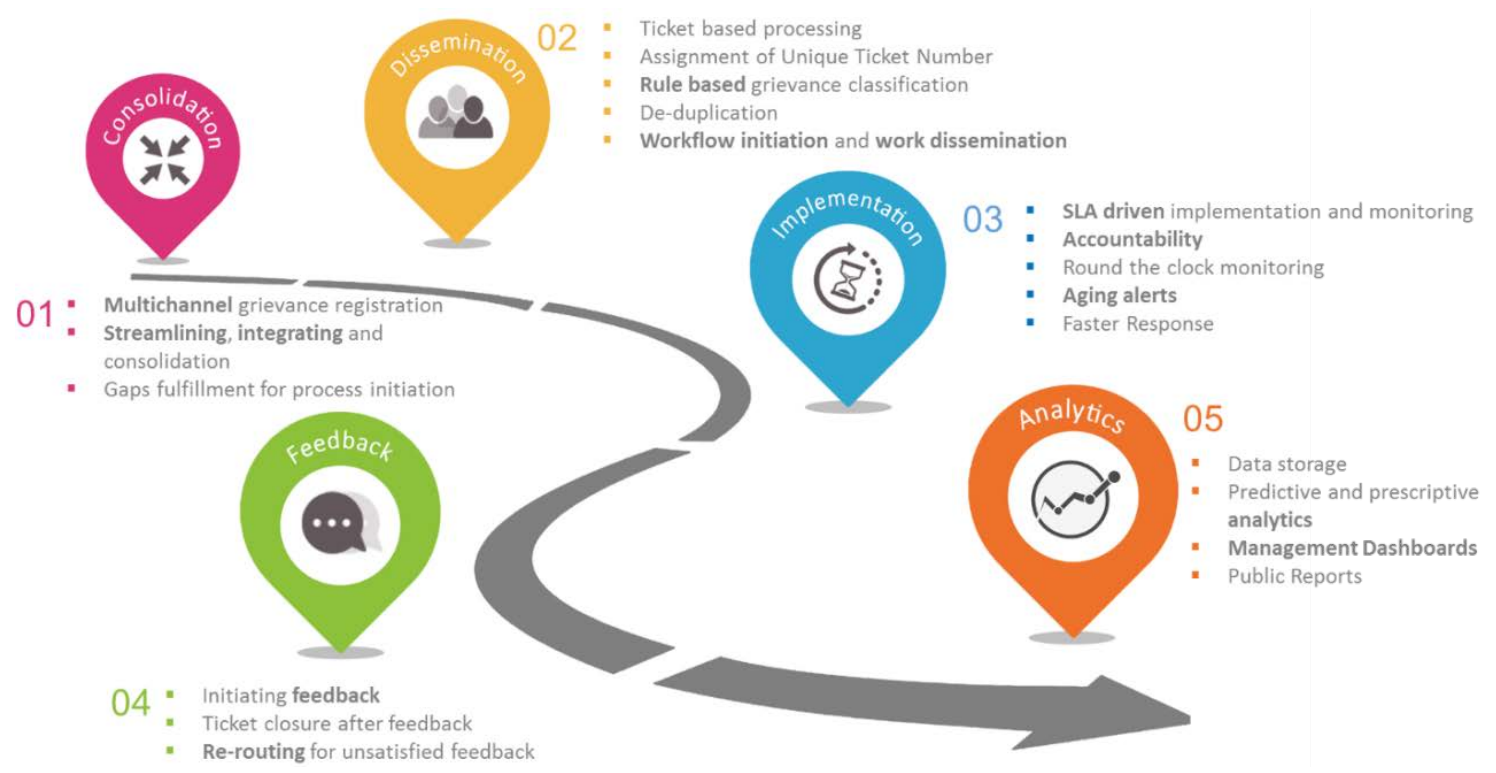

Figure 3. Components of iPGRS

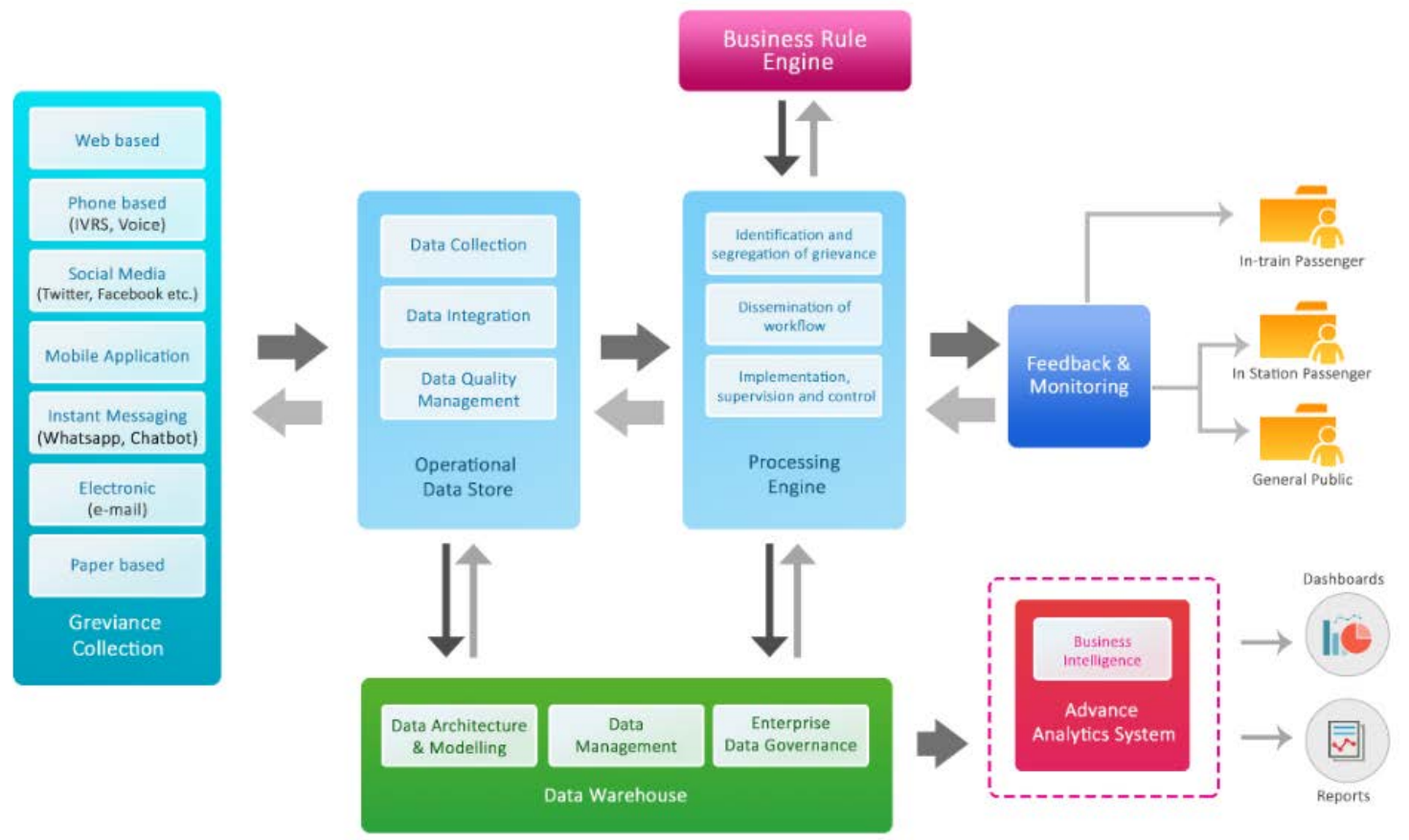

Figure 4. A system overview of iPGRS 


\section{Unique Features of iPGRS}

The iPGRS has many standout and unique features as depicted in Figure 5.

- Multichannel - iPGRS is a next-gen grievance redress mechanism with multichannel complaint handling capabilities, a capability that not many railway operators currently possess. Not only does the system capture information from any channel paper, phone, mobile app, social media, instant messaging, and online, it also integrates them all in a single place. Moreover, the system is also geared to integrate any channels that may emerge in the future, such as chatbots and robots.

- $\quad$ Flexible and scalable - Being a highly flexible and scalable solution, iPGRS is capable of handling almost any volume of complaints.

- $\quad 24 x 7$ availability - It is always at work, accepting and disseminating complaints at any time of day or night.

- $\quad$ Alerts and notifications - The system maintains two-way communication with passengers throughout the grievance redress lifecycle, notifying them of all important events such as the raising of a service request, initiation of action, closure, and so on.

- $\quad$ Real-time and offline - iPGRS gets into action the moment a complaint is received, moving information to the concerned departments without delay. In the event that a particular operating crew member lacks Internet connectivity, the system passes on the complaint information offline - to the phone or another offline communication mode.
- $\quad$ Data storage and analytics - The system has robust data storage and analytical capabilities.

\section{How a Railway Can Benefit from iPGRS}

iPGRS offers several advantages to railway companies as summarized below:

- Enhanced Customer Experience: It improves customer experience by empowering railway crews to respond faster to passenger grievances based on features such as integrated process-driven operations and real-time track-and-trace visibility.

- Improved Process Efficiency: iPGRS eliminates cumbersome, unsystematic and old processes to bring in process automation and free up crew time.

- Better Inter-organizational Workforce Communication: It ensures uninterrupted access to relevant information by seamlessly updating the company and crew members.

- Greater Operational Visibility: With iPGRS, the company achieves a high level of operational visibility with an integrated workflow and ticket-based system. This brings greater accountability, transparency and efficiency to the complaint handling process.

- Increased Service Levels: iPGRS enhances service quality by identifying and mitigating pain points in railway operations, and improves passenger services through advanced analytics.

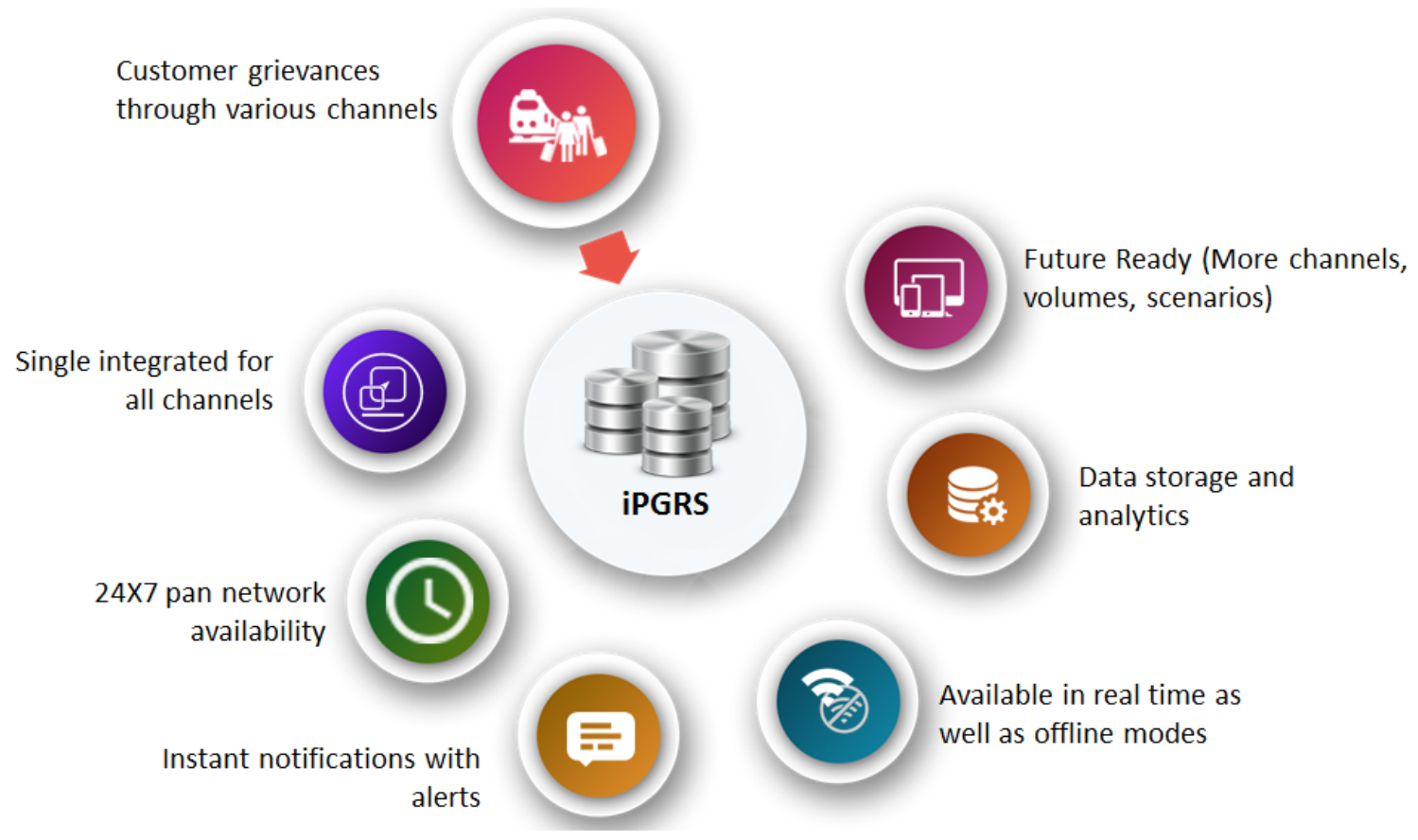

Figure 5. Unique Features of iPGRS 


\section{Conclusions}

Research has found that across product and service categories, 52 percent of consumers switched providers in the preceding twelve months due to poor customer service [10]. With rail transport usage set to grow in most markets, railway operators must find a way to deliver great service and experiences, just like their competitors, including airlines and new-age transportation providers. The quality of complaint resolution has a huge bearing on the quality of service. Today's passengers are digitally empowered to vocalize their grievances in real-time and expect the resolution to be immediate, painless and empathetic. Unfortunately, current complaint handling mechanisms suffer from several weaknesses-lack of multichannel capability, poor visibility and an ineffective feedback mechanism, being some of them-that delay resolutions and cause dissatisfaction among passengers. As a result, there is a clear need for a robust passenger grievance redress mechanism with multichannel integration capabilities, scalability, a feedback mechanism and powerful analytics. iPGRS is a "best-fit" solution that meets all these expectations. A prototype was developed and demonstrated to a large railway organization in Asia. This solution is under consideration.

\section{REFERENCES}

[1] Global rail passenger traffic from 2011 to 2016, by region, Statista
[2] Global rail traffic forecast for continued growth, International Railway Journal, http://www.railjournal.com/i ndex.php/metros/global-rail-traffic-forecast-for-continued-g rowth.html

[3] Assessing the global transport infrastructure market: Outlook to 2025, PwC,https://www.pwc.com/gx/en/transpo rtation-logistics/pdf/assessing-global-transport-infrastructur e-market.pdf

[4] Clean Rail Transportation Options, Springer, http://www.springer.com/in/book/9783319217253

[5] https://www.arup.com/-/media/arup/files/publications/.../aru p_future_of_rail_2050.pdf

[6] Improving customer satisfaction in the airline industry lessons from Southwest Airlines, Telus International, https://telusinternational-europe.com/customer-satisfactionairline-industry-southwest-airlines/

[7] Airlines in search of customer loyalty need to do more than improve service, Help Scout, https://www.helpscout.net/blo g/airline-customer-service/

[8] You asked, they answered: How Southwest Airlines does social customer service, https://blogs.oracle.com/socialspotl ight/you-asked,-they-answered:-how-southwest-airlines-doe s-social-customer-service

[9] UK rail passengers 'resigned to bad service', Evening Standard, https://www.standard.co.uk/news/transport/uk-rail-passenge rs-resigned-to-bad-service-a3467116.html

[10] Customer Loyalty: 20 compelling reasons to double down on it, Fieldbloom, https://www.fieldboom.com/blog/customer-loyalty-infogra phic/ 